\title{
Pairs of Creatine Kinase Serum Activity
}

\author{
Dirk Moßhammer ${ }^{1, *}$, Rainer Muche $^{2}$, Dagmar Menzel $^{3}$, Christina Ring $^{2}$, Dorothee Wernet ${ }^{3}$, \\ Christoph Meisner ${ }^{4}$, Christoph H. Gleiter ${ }^{5}$, Gernot Lorenz ${ }^{1}$ and Klaus Mörike ${ }^{5}$
}

${ }^{1}$ Division of General Medicine, University of Tübingen; ${ }^{2}$ Institute of Biometrics, University of Ulm; ${ }^{3}$ Institute for Clinical and Experimental Transfusion Medicine, University Hospital of Tübingen; ${ }^{4}$ Institute of Medical Biometrics, University Hospital of Tübingen and ${ }^{5}$ Institute of Pharmacology and Toxicology, Department of Clinical Pharmacology, University Hospital of Tübingen

\begin{abstract}
Background: For patients with an increased risk of developing myopathy with statin treatment, guidelines recommend to measure CK before and 6-12 weeks after initiating statin treatment. Further, in clinical trials of statins, the number of patients experiencing elevated creatine kinase (CK) serum activity is commonly reported as a safety parameter. Usually exceeding the upper limit of normal (ULN) is used as the basis of description to characterize muscular damage potentially caused by statins. Therefore, basic data on both, proportions of patients with specific CK increases, and absolute and relative CK increases, would be helpful to assess potential CK chances in patients. However, no such data are available so far.

Methods: CK activity was measured in a group of blood donors $(\mathrm{n}=40)$ at two occasions 10 weeks apart. The $95^{\text {th }}$ percentile of absolute and relative CK increases was selected to separate true from irrelevant changes. A CK difference was defined to be true if a random measurement error of $10 \%$ was exceeded. The numbers of subjects with true CK differences were characterised by both measurements being under, crossing, or being above ULN, and twice ULN, respectively.

Results: Five percent of subjects $\left(95^{\text {th }}\right.$ percentile) had an increase of CK more than $139 \%$ (men) or $120 \%$ (women), respectively, as compared with the first measurement. Five percent of subjects had an absolute difference of at least 94 U/1 (men) and $16 \mathrm{U} / 1$ (women), respectively. Thirty-one percent of subjects had both CK activities below 2 times the ULN. Six percent of them had the first measurement under and the second above ULN. In three percent, the first measurement was under and the second above 2 times the ULN. In no subject, the first and the second measurement was above 2 times the ULN.

Conclusion: It is concluded that CK serum activity substantially varies between two measurements. The present data provide the basis for developing reference ranges of CK activity pairs by simple variables that can be useful for practitioners and trialists.
\end{abstract}

Key Words: Lipid-lowering therapy, myopathy, statins, creatine kinase.

\section{INTRODUCTION}

In statin therapy, efforts need to be directed to protect patients from myopathy. Patients at risk to develop myopathy should be identified at an early stage. Therefore, experts recommend to measure CK serum activity before and after initiating statin therapy in patients at risk [1]. If muscle complaints arise during treatment, $\mathrm{CK}$ analysis should be repeated after 6 to 12 weeks [2]. Although reference ranges for single-time CK serum activities have been established, no such data on intra-individual CK pairs are available.

We therefore aimed to characterise variation of CK pairs in healthy blood donors. These data should subsequently serve to establish reference ranges of CK pairs in patients starting statin therapy.

*Address correspondence to this author at the Division of General Medicine, University Tübingen, Keplerstrasse 15, D-72074 Tübingen, Germany; Tel: +49 707129 80255; Fax: +49 707129 5896;

E-mail: dirk.mosshammer@uni-tuebingen.de

\section{MATERIALS AND METHODOLOGY}

\section{Participants}

Blood donors of a university-based public blood donation center participated in this project. Inclusion criteria were age $>40$ years (because statins are rarely used in younger adults) and informed consent. Exclusion criteria, based on their potential to be associated with CK increase, were alcohol abuse during the previous 3 days, or history of alcoholism, asthma attack during the previous 3 days, or cough attack, seizure during the previous 3 days, massages during the previous 3 days, hypertensive crisis, intramuscular injection during the previous 7 days, known liver disease, muscle ache, myocardial infarction during the previous 6 months, percutaneous coronary intervention during the previous 6 months, blunt trauma, muscle disease (myositis, polymyositis, dermatomyositis, progressive muscular dystrophy Duchenne, congenital myopathy), surgical procedures during the previous 6 months, serious disease, malignant disease or chemotherapy, heavy physical exercise during the previous 3 days (see 
below), use of statins or corticosteroids. These criteria were part of the informed consent. Data on ethnic background, muscle ache, drug use, body weight and height, and massages, sports or physical activity (definition scale: $0=$ no sport or physical activity, $1=$ light sport or physical activity, e.g., walking, 2 = medium sport or physical activity, e.g., aerobic, jogging, 3 = heavy sport or physical activity, e.g., competitional sport, during the previous 3 days) were collected using a separate questionnaire. The ethical committee of the Medical Faculty of the University of Tübingen approved the study protocol.

\section{Analysis}

CK serum activity was measured on two occasions (CK1 and CK2), about 10 weeks apart, in each subject. All tests were performed in one laboratory, using the modified method by Szasz using the ADVIA ${ }^{\circledast} 1650$ Chemistry Systems Bayer ${ }^{\otimes}$ kit. The CK assay used was the standard $37^{\circ} \mathrm{C}$ IFCC (International Federation of Clinical Chemistry) method. The upper limit of normal (ULN) was $170 \mathrm{U} / \mathrm{l}$ (women) and $190 \mathrm{U} / \mathrm{l}$ (men), respectively.

A random error of $10 \%$ was assumed, based on the CK measurement method's coefficient of variation (CV) of $3 \%$ and a resulting confidence range $(=3 \mathrm{CV})$ of about $10 \%$.

Target variables: The following target variables were described:

- 95th percentile of the absolute differences (CK2-CK1) and

- 95th percentile of the relative differences ((CK2/CK1) x $100 \%)$.

Values exceeding these limits were considered abnormal. No parametric distribution was assumed for CK. $90 \%$ confidence intervals $(\mathrm{CI})$ were calculated using the percentile method with bootstrap samplings $(n=1000$, a commonly used sample size) [3]. Additional target variables were defined using the following limits (Table $\mathbf{3}$ ): upper limit of normal (ULN), 1.1-fold of first value (taking $3 \mathrm{CV}$ into account), and twice ULN (based on arbitrary limits found in published reports, e.g. $[4,5])$.

Nonparametric $90 \%$ confidence intervals were determined for proportions of subjects meeting these criteria. Bootstrap sampling and data analyses were performed with the $\mathrm{SAS}^{\circledast}$ software version 9.1. Tests for confounding (age, sex, body mass index, physical activity) were performed using Wilcoxon rank sum tests for unpaired samples and Chi square tests, respectively. All tests were done in an exploratory fashion. The significance level was set to $5 \%$.

\section{RESULTS}

40 blood donors met the inclusion criteria. Four male donors reporting heavy sport or muscle ache were excluded. All 36 donors were of Caucasian ethnic background. Seven participants reported using drugs, i.e. four women ( 1 contraceptive, 2 angiotensin-converting enzyme inhibitor, 1 thyroxin) and three men (thyroxin, beta blocker, angiotensin receptor blocker), see Table 1.

CK serum activity pairs and the target variables are shown in Table 2. Differences greater than $94 \mathrm{U} / \mathrm{l}$ (men) or $16 \mathrm{U} / 1$ (women), respectively, and deviations of CK2 in relation to CK1 of more than $139 \%$ (men) or $120 \%$ (women) were found to occur in $5 \%$ of subjects.

Fig. (1) shows the distribution of the individual CK activity difference values (CK2-CK1). An outlier is observed among the male group.

Table 3 shows the absolute and relative frequencies of subjects whose CK values met the logical operations (random error of $10 \%$, once or twice ULN). $25 \%$ of donors had significant variations within the reference range, and $11 \%$ of subjects had such variations above ULN.

Table 1. Demographic Data and Time Interval between CK Measurements

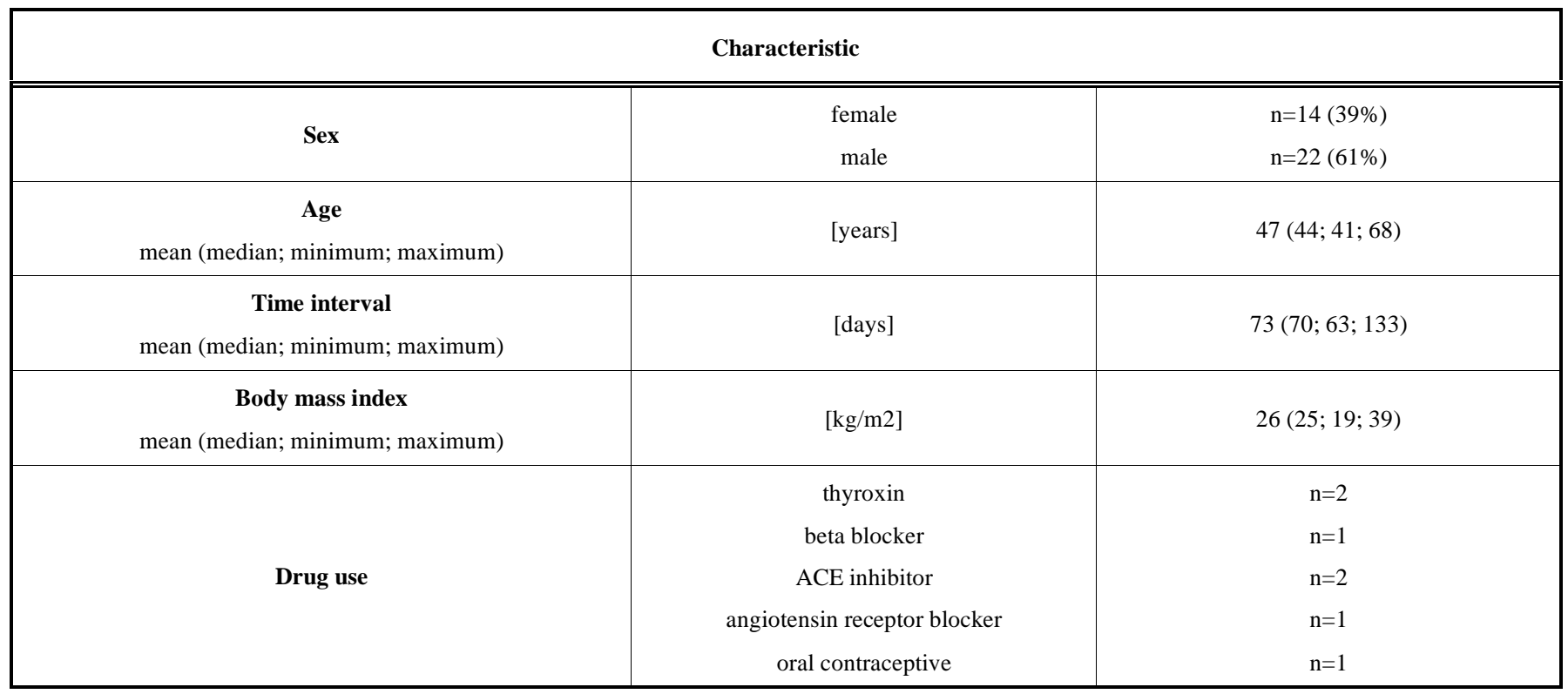


Table 2. The $95^{\text {th }}$ Percentile $\left(Q_{95}\right)$ of Creatine Kinase (CK) Serum Activities of First and Second Measurement (CK1 and CK2) and the Target Variables (CK2-CK1) and (CK2/CK1)x100* with 90\% $\mathrm{CI}^{\dagger}(\mathrm{n}=36)$

\begin{tabular}{|c|c|c|c|c|}
\hline \multicolumn{5}{|c|}{ Total cohort } \\
\hline $\mathbf{Q}_{95}$ & $257[238,377]$ & $348[229,796]$ & $47[38,419]$ & $137[128,211]$ \\
\hline \multicolumn{5}{|c|}{ Men } \\
\hline \multicolumn{5}{|c|}{ Women } \\
\hline $\mathbf{Q}_{95}$ & $196[182,238]$ & $176[146,215]$ & $16[13,17]$ & $120[115,125]$ \\
\hline
\end{tabular}

Legend: $*((\mathrm{CK} 2) /(\mathrm{CK} 1)) \times 100 \%$, i.e. percent of CK1. $\dagger$ determination of CI with the percentile method using bootstrap samplings (n=1000) [3]. Values of the target variables are bold.

Table 3. Proportions of Patients with Specific CK Increases (Including CI $I^{\dagger}$ ) Referred to the ULN ${ }^{\S}$ and 2 times the ULN

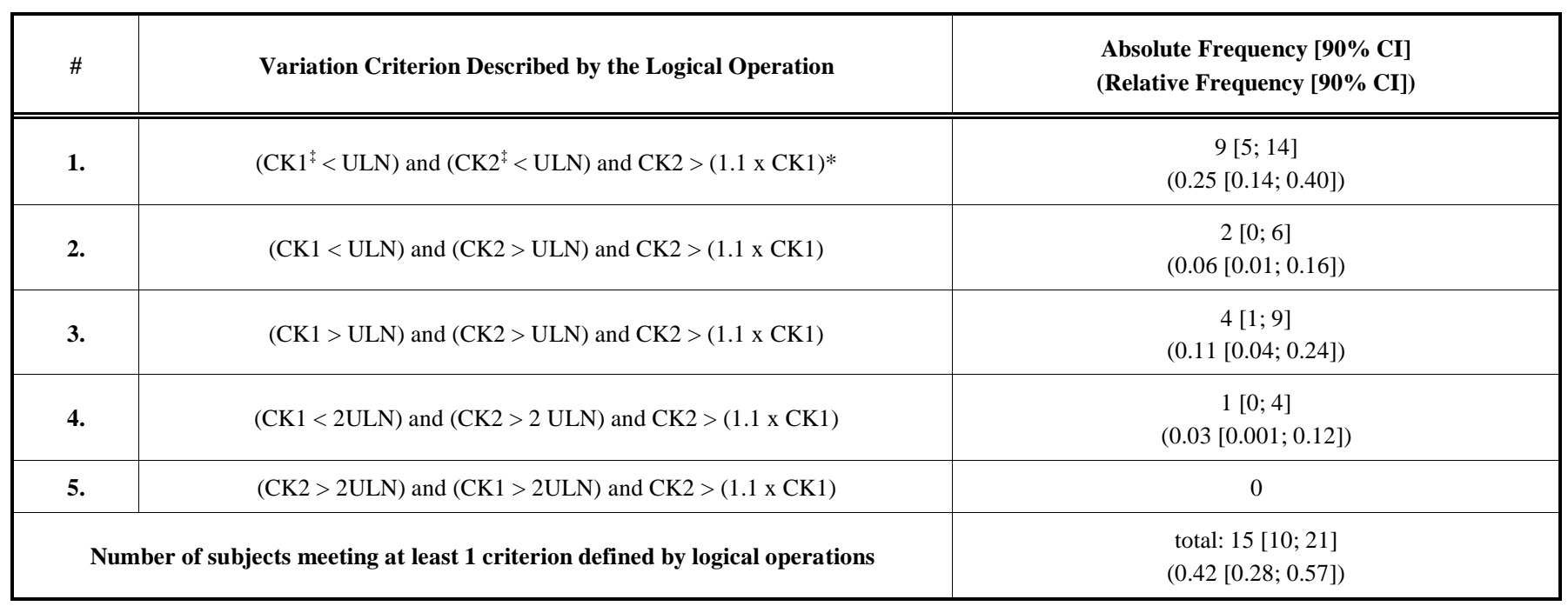

Legend: *: The coefficient of variation (CV) of this measurement is about $3 \%$, and thus confidence range $(=3 \mathrm{CV})$ about $10 \%$. A true difference was assumed if $>10 \%$ deviation. ${ }^{\dagger} \mathrm{CI}$ : Confidence interval. ${ }^{\ddagger} \mathrm{CK} 1$ und CK2: first and second CK measurement, respectively. ${ }^{8} \mathrm{ULN}$ : Upper limit of normal (i.e., females $170 \mathrm{U} / 1$, males $\left.190 \mathrm{U} / \mathrm{l}\right)$.

Explanation of \#1: Twenty five percent of subjects meet the first variation criterion by having CK values below the ULN, but by having the second measurement 1.1 times greater than the first one (taking into account the random measurement error of $10 \%$, see above).

Explanation of \#2: Six percent of subjects had their first CK value below and the second above the ULN (but below 2 times the ULN) considering the random measurement error of $10 \%$.

Explanation of \#3: Eleven percent of subjects had their first and second CK value above the ULN considering the random measurement error of $10 \%$.

Explanation of \#4: In 3\% of subjects, the first CK value is below double ULN and the second is above double ULN regarding the random measurement error of $10 \%$ ( $3 \mathrm{CV}$ ).

Testing for potential confounders (age, sex, body mass index, physical activity), sex gives a borderline significant association $(\mathrm{p}=0.05)$ : Only 3 out of 15 subjects meeting at least 1 variation criterion were women.

\section{DISCUSSION}

Using pre-specified criteria, the present data show that individual pairs of $\mathrm{CK}$ serum activities are variable. On the basis of these variation criteria and results it seems appropriate to establish reference ranges for two consecutive CK measurements in individuals. For the outlier of individual CK differences (Fig. 1), no obvious reason (such as age, body mass index, physical activity, medication) was found. Recent data indicate that inter-individual variability of $\mathrm{CK}$ activity in the general population is greater than previously suggested and that relatively high values frequently occur in different subgroups [6]. Those and our findings are consistent with the existing knowledge that males have higher values than females, as reflected by higher ULN than in females. However, higher CK activity levels per se are not sufficient to explain the higher intra-individual CK activity differences that we observed. Whatever the underlying reason may be, the existence of outstanding CK activity pairs will be important for subsequent studies evaluating $\mathrm{CK}$ alterations upon statin treatment initiation.

Our data do not allow conclusions about the clinical significance of CK alterations. Rather, our data will provide the basis for prospective studies to address the prognostic value of CK alterations and, specifically, minimum changes required to stop treatment. 


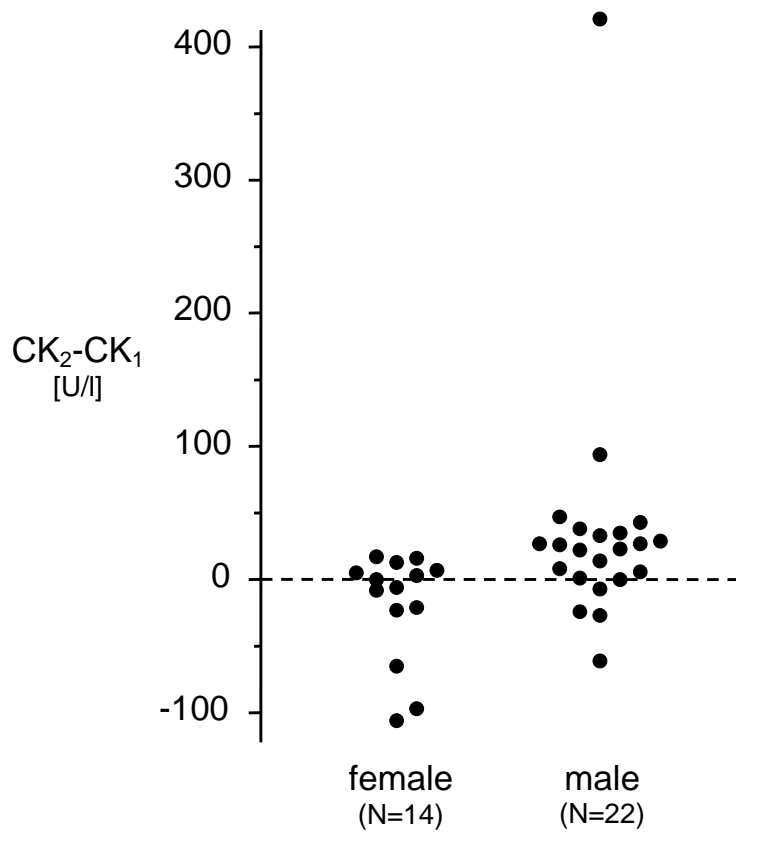

Fig. (1). Distribution of the CK activity difference values among the male and female group.

Physicians referring to recommendations to measure CK before and 10 weeks after initiation of statin treatment in patients at myopathy risk will benefit from such reference ranges. Furthermore, such reference ranges may be included in future statin trials using $\mathrm{CK}$ as a safety parameter.

\section{CONCLUSION}

It is concluded that $\mathrm{CK}$ serum activity substantially varies between two measurements. The present data provide the basis for developing reference ranges of CK activity pairs by simple variables.

\section{ACKNOWLEDGEMENT}

We thank Christiane Hügel (Institute of Medical Biometrics, University Hospital of Tübingen) for SAS macroprogramming.
ABBREVIATIONS
CK
$=$ Creatine kinase
$\mathrm{CK} 1$ and $\mathrm{CK} 2=$ First and second CK measurement, respectively
$\mathrm{CI} \quad=$ Confidence interval
$\mathrm{CV}=$ Coefficient of variation
HMG CoA = Hydroxy-methyl-glutaryl coenzyme A
ULN $=$ Upper limit of normal

\section{REFERENCES}

[1] McKenney, J.M.; Davidson, M.H.; Jacobson, T.A.; Guyton, J.R. Final conclusions and recommendations of the National Lipid Association Statin Safety Assessment Task Force. Am. J. Cardiol., 2006, 97(8A), 89C-94C.

[2] Pasternak, R.C.; Smith, S.C. Jr.; Bairey-Merz, C.N.; Grundy, S.M.; Cleeman, J.I.; Lenfant, C. ACC/AHA/NHLBI clinical advisory on the use and safety of statins. J. Am. Coll. Cardiol., 2002, 40(3), 567-572.

[3] Campbell, M.J.; Daly, L.E.; Machin, D. In Statistics with confidence; Special Topics, 2nd ed.; British Medical Journal Books: Bristol, 2000, pp. 153-171.

[4] Bruckert, E.; Hayem, G.; Dejager, S.; Yau, C.; Begaud, B. Mild to moderate muscular symptoms with high-dosage statin therapy in hyperlipidemic patients - the PRIMO study. Cardiovasc. Drugs Ther., 2005, 19(6), 403-414.

[5] Ellen, R.L.; McPherson, R. Long-term efficacy and safety of fenofibrate and a statin in the treatment of combined hyperlipidemia. Am. J. Cardiol., 1998, 81(4A), 60B-65B.

[6] Brewster, L.M.; Mairuhu, G.; Sturk, A.; van Montfrans, G.A. Distribution of creatine kinase in the general population: implications for statin therapy. Am. Heart J., 2007, 154(4), 655-661. 